01

\title{
Диссипативное туннелирование электронов в вертикально связанных двойных асимметричных квантовых точках InAs/GaAs(001)
}

\author{
() М.Б. Семенов, ${ }^{1}$ В.Д. Кревчик, ${ }^{1}$ Д.О. Филатов, ${ }^{3}$ А.В. Шорохов, ${ }^{1,2,7,8}$ А.П. Шкуринов, ${ }^{1,4}$ И.А. Ожередов, ${ }^{1,4}$ \\ П.В. Кревчик, ${ }^{1}$ Y.H. Wang, ${ }^{5}$ T.R. Li, ${ }^{5}$ A.K. Malik, ${ }^{6}$ M.O. Марычев, ${ }^{3}$ H.B. Байдусь, ${ }^{3}$ И.М. Семенов ${ }^{1}$ \\ ${ }^{1}$ Пензенский государственный университет, \\ 440026 Пенза, Россия \\ ${ }^{2}$ University of Jyväskylä, Seminaarinkatu, \\ 15, PO BOX 35, Fl-40014, Finland \\ ${ }^{3}$ Нижегородский государственный университет им. Н.И. Лобачевского, \\ 603950 Нижний Новгород, Россия \\ ${ }^{4}$ Институт проблем лазерных и информационных технологий РАН, \\ 140700 Шатура, Московская обл., Россия \\ ${ }^{5}$ Key Laboratory for Special Function Materials, School of Physical Science and Technology, Lanzhou University, \\ 730000 Lanzhou, China \\ ${ }^{6}$ Department of Physics, Multanimal Modi College Modinagar, \\ 201204 Uttar Prasesh, India \\ ${ }^{7}$ Мордовский государственный университет им. Н.П. Огарева, \\ 430005 Саранск, Россия \\ ${ }^{8}$ International Research Centre Mag Top, Institute of Physics, Polish Academy of Sciences, \\ Aleja Lotnikow 32/46, PL-02668 Warsaw, Poland \\ e-mail: Misha29.02.1@gmail.com
}

Поступило в Редакцию 18 марта 2021 г.

В окончательной редакции 18 марта 2021 г.

Принято к публикации 18 марта 2021 г.

\begin{abstract}
Приведены результаты экспериментальных исследований фотоэлектрических свойств GaAs $p-i-n$-фотодиода с двойными асимметричными квантовыми точками (ДАКТ) InAs, полученными методом самоформирования в процессе МOC-гидридной эпитаксии. В зависимости фототока от напряжения обратного смещения при монохроматическом фотовозбуждении ДАКТ на длине волны, соответствующей энергии межзонных оптических переходов между основными состояниями дырок и электронов в квантовых точках (КТ) большего размера, обнаружены 3 пика, связанные с туннелированием фотовозбужденных электронов между КТ, в том числе - диссипативным (с поглощением и испусканием оптических фононов). Результаты эксперимента качественно согласуются с теоретической полевой зависимостью вероятности $1 D$-диссипативного туннелирования между КТ.
\end{abstract}

Ключевые слова: вертикально-совмещенные двойные асимметричные квантовые точки, InAs, GaAs, туннельные оптические переходы, фотопроводимость, диссипативное туннелирование.

DOI: $10.21883 / J T F .2021 .10 .51354 .66-21$

\section{Введение}

Экспериментальное наблюдение теоретически предсказываемых макроскопических эффектов диссипативного туннелирования [1-12] является одной из важнейших задач в указанной предметной области $[1,7,11]$. В последнее десятилетие авторами настоящей работы экспериментально наблюдались эффекты, обусловленные диссипативным туннелированием электронов в ряде искусственных нанорамерных систем. Достигнутые на сегодняшний день результаты обобщены в табл. 1. В настоящей работе экспериментально исследована зависимость фоточувствительности (ФЧ) $p-i-n$-фотодиода на основе GaAs c двуслойным массивом самоорганизующихся двойных асимметричных квантовых точек (ДАКТ) InAs при резонансном фотовозбуждении межзонных оптических переходов между основным элек- тронным и дырочным состояниями в более крупных квантовых точках (КТ) с последующим диссипативным туннелированием электронов на основное состояние в КТ меньшего размера. Результаты экспериментов интерпретированы на основе теории одномерного диссипативного туннелирования с учетом влияния двух локальных фононных мод матрицы GaAs (рассматриваемой как термостат) $[11,12]$.

\section{1. Экспериментальная часть}

\section{1. Формирование $p-i-n$-структур с массивами ДАКТ InAs/GaAs(001) и меза-фотодиодов на их основе}

Диодная GaAs $p-i-n$-структура со встроенными массивами ДАКТ InAs была выращена на подложке 
Таблица 1. Экспериментально наблюдаемые эффекты диссипативного туннелирования

\begin{tabular}{|c|c|c|c|}
\hline $1 D$ & $1 D$ & $2 D$ & $2 D$ \\
\hline Предел слабой диссипации & Предел сильной диссипации & Предел слабой диссипации & Предел сильной диссипации \\
\hline $\begin{array}{l}\text { Максимум на } \\
\text { туннельных ВАХ } \\
\text { для наночастиц } \mathrm{Au} \\
\text { в пленках } \mathrm{SiO}_{2}[11]\end{array}$ & $\begin{array}{l}\text { Серия неэквидистантных } \\
\text { пиков на туннельных } \\
\text { ВАХ КТ } \\
\text { InAs/GaAs(001) [12] }\end{array}$ & $\begin{array}{l}2 D \text {-бифуркации на } \\
\text { туннельных ВАХ } \\
\text { массивов наночастиц } \mathrm{Au} \\
\text { в пленках } \mathrm{SiO}_{2}[11]\end{array}$ & $\begin{array}{l}2 D \text {-бифуркации на } \\
\text { полевой зависимости ФЧ } \\
p-i-n \text {-структур } \\
\text { с ДАКТ InAs/GaAs }(001)\end{array}$ \\
\hline
\end{tabular}

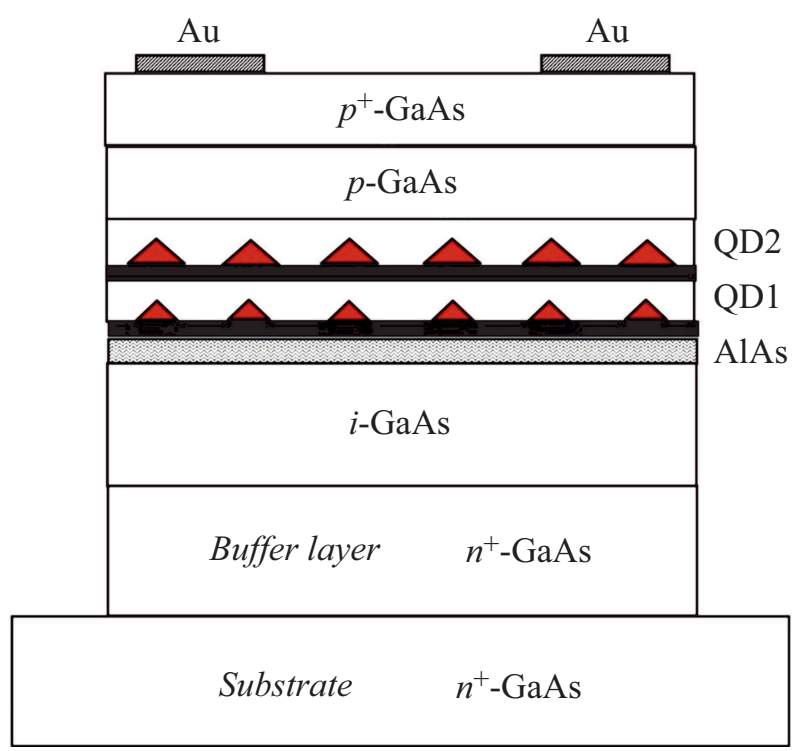

Рис. 1. Схема меза-фотодиода на базе GaAs $p-i-n$-структуры с массивом вертикально-сдвоенных ДАКТ InAs/GaAs(001).

$n^{+}-\mathrm{GaAs}(001)$ фирмы „MeGa SM“, разориентированной на $2^{\circ}$ по направлению $(011)$, методом МОС-гидридной эпитаксии при пониженном давлении (100 mbar) на установке Aixtron AIX 200RF. В качестве прекурсоров основных компонентов структур использовались $\mathrm{Ga}\left(\mathrm{CH}_{3}\right)_{3}, \operatorname{In}\left(\mathrm{CH}_{3}\right)_{3}$ и $\mathrm{AsH}_{3}$. Схема $p-i-n$-структуры показана на рис. 1. На поверхности подложки $n^{+}$$\mathrm{GaAs}(001)$ выращивался буферный слой $n^{+}$-Ga толщиной $300 \mathrm{~nm}$, легированный $\mathrm{Si}$ из $\mathrm{SiH}_{4}$. Затем выращивался слой специально не легированного $i$-GaAs (фоновая концентрация электронов $n_{0} \sim 5 \cdot 10^{15} \mathrm{~cm}^{-3}$ ) толщиной $\approx 350 \mathrm{~nm}$. На поверхности слоя $i$-GaAs методом самоформирования по механизму Странски-Крастанова [13] при температуре роста $T_{g} \approx 490^{\circ} \mathrm{C}$ формировался массив KT InAs (KT1) высотой $h \approx 4 \mathrm{~nm}$ и латеральными размерами $D=12-14 \mathrm{~nm}$. Предварительно с целью стимуляции самоформирования КT InAs на поверхность слоя $i$-GaAs осаждался подслой AlAs толщиной $\approx 0.8 \mathrm{~nm}$. Квантовые точки InAs заращивались спейсором $i$-GaAs толщиной $8 \mathrm{~nm}$, на поверхности которого при $T_{g} \approx 505^{\circ} \mathrm{C}$ формировался второй слой КТ InAs $($ КT2) с $D=16-18 \mathrm{~nm}$ и $h \approx 6 \mathrm{~nm}$. Поверх слоя
КТ2 выращивался слой $i$-GaAs толщиной $\approx 7 \mathrm{~nm}$, а затем - слой $p$-GaAs толщиной $\approx 190 \mathrm{~nm}$. На поверхность $p$-GaAs наносился подконтатный слой $p^{+}$-GaAs: C толщиной $\approx 60 \mathrm{~nm}$. Кроме $p-i-n$-структуры с ДАКТ $\mathrm{InAs} / \mathrm{GaAs}(001)$, был выращен образец-спутник на подложке полуизолирующего $\mathrm{GaAs}(001)$, содержавший массив ДАКТ, выращенных в тех же условиях, встроенных в толщу слоя $i$-GaAs (толщина буферного слоя была $500 \mathrm{~nm}$, покровного слоя $-200 \mathrm{~nm})$. Данный образец предназначался для исследования энергетического спектра ДАКТ методом спектроскопии фотолюминесценции (ФЛ).

На базе $p-i-n$-структуры с массивом ДАКТ $\mathrm{InAs} / \mathrm{GaAs}(001)$ методом фотолитографии с жидкостным химическим травлением были сформированы мезафотодиоды с мезами цилиндрической формы диаметром $500 \mu \mathrm{m}$ с кольцевым верхним омическим контактом.

\section{2. Исследование энергетического спектра ДAKT InAs/GaAs(001)}

Энергетический спектр ДАКТ $\operatorname{InAs} / \mathrm{GaAs}(001)$ определялся методом спектроскопии ФЛ. Измерения ФЛ проводились при 77 и $300 \mathrm{~K}$ при помощи монохроматора МДР-23 с возбуждением $\mathrm{He}-\mathrm{Ne}$-лазера с длиной волны излучения $\lambda 632.8 \mathrm{~nm}$ и мощностью $30 \mathrm{~mW}$ и $\mathrm{Ge}$ фотодетектора с синхронным детектированием.

На рис. 2 представлены спектры ФЛ $p-i-n$-структуры с ДАКТ InAs/GaAs(001) и образца-спутника при 77 и $300 \mathrm{~K}$. В спектрах ФЛ кроме пика краевой ФЛ GaAs (энергия максимума $h v_{m} \approx 1.42$ и $\approx 1.51 \mathrm{eV}$ при 300 и $77 \mathrm{~K}$ соответственно) наблюдались пики в области $0.9 \mathrm{eV}<h v<1.4 \mathrm{eV}$, связанные с межзонными рекомбинационными излучательными оптическими переходами в KT InAs. Пики с $h v_{m} \approx 1.05-1.1 \mathrm{eV}$ и $\approx 1.2-1.25 \mathrm{eV}$ при $77 \mathrm{~K}$ связаны с межзонными переходами между основными размерно-квантованными состояниями электронов и дырок в КТ2 и КТ1 соответственно. На основе расчетов энергий размерно-квантованных состояний в KT $\operatorname{InAs} / \operatorname{GaAs}(001)$, имеющих форму пирамиды, ограненной плоскостями (101) [14], из значений $h v_{m}$ были оценены геометрические параметры КТ, а также энергии основных состояний электронов и дырок в КТ $E_{e 000}$ и $E_{h 000}$ соответственно (табл. 2). 

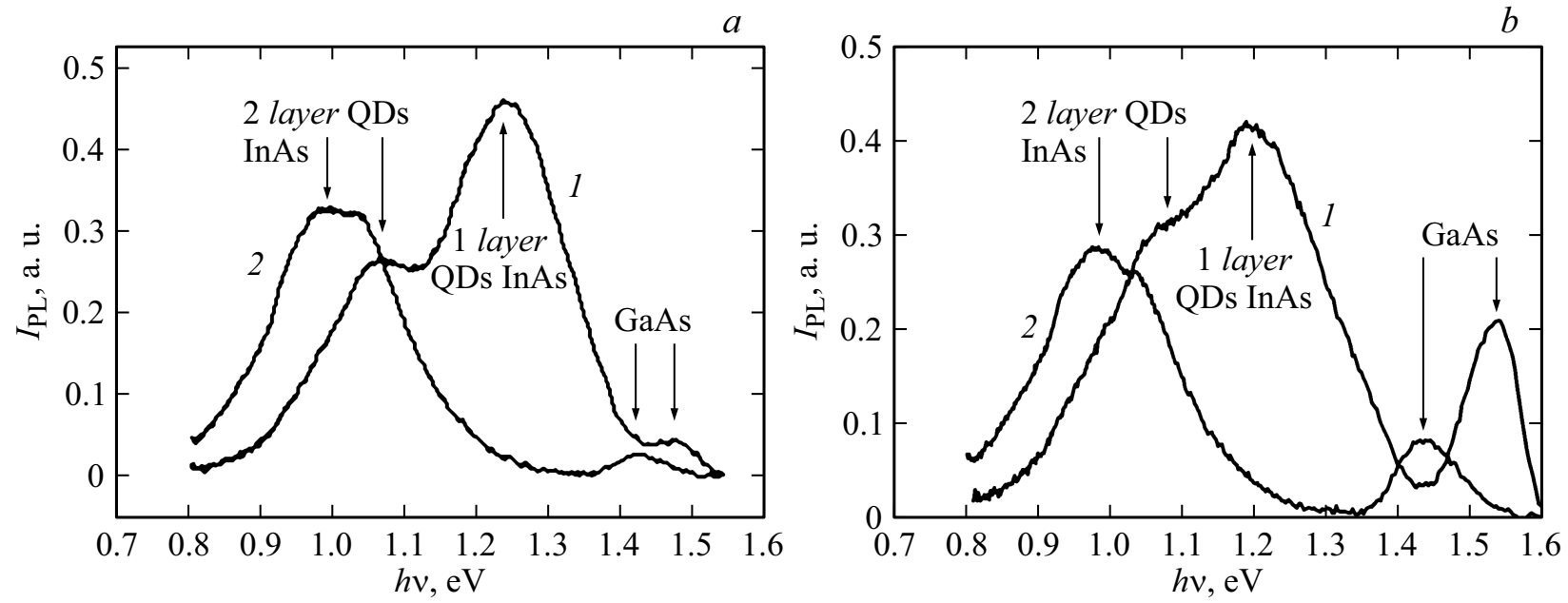

Рис. 2. Спектры ФЛ $p-i-n$-структуры с ДАКТ $\operatorname{InAs} / \mathrm{GaAs}(001)(a)$ и образца-спутника на полуизолирующей подложке $(b)$ при $77 \mathrm{~K}(1)$ и $300 \mathrm{~K}(2)$.

Таблица 2. Параметры ДАКТ InAs/GaAs(001), определенные из спектров ФЛ

\begin{tabular}{c|c|c|c}
\hline Параметр & $\begin{array}{c}\text { Единицы } \\
\text { измерения }\end{array}$ & КТ1 & КТ2 \\
\hline$D$ & $\mathrm{~nm}$ & $12-14$ & $16-18$ \\
$h$ & $\mathrm{~nm}$ & $4 \pm 1$ & $6 \pm 1$ \\
$h v_{m}(77 \mathrm{~K})$ & $\mathrm{eV}$ & 1.203 & 1.047 \\
$h v_{m}(300 \mathrm{~K})$ & $\mathrm{eV}$ & 1.18 & 0.95 \\
$\lambda_{m}(77 \mathrm{~K})$ & $\mu \mathrm{m}$ & 1.031 & 1.184 \\
$\lambda_{m}(300 \mathrm{~K})$ & $\mu \mathrm{m}$ & 1.05 & 1.31 \\
$\Delta E_{e 000}$ & $\mathrm{meV}$ & 161 & 266 \\
$E_{e 000}(77 \mathrm{~K})$ & $\mathrm{meV}$ & 439 & 334 \\
$\Delta E_{h 000}(77 \mathrm{~K})$ & $\mathrm{meV}$ & 137 & 189 \\
$E_{h 000}(77 \mathrm{~K})$ & $\mathrm{meV}$ & 263 & 211
\end{tabular}

Там же приведены длина волны излучения, соответствующего межзонному переходу между основными состояниями электронов и дырок $\lambda_{m}$, а также высота эмиссионного барьера для электронов и дырок в КТ $\Delta E_{e 000}$ и $\Delta E_{h 000}$ соответственно. На основании этих данных были рассчитаны равновесные зонные диаграммы исследуемой $p-i-n$-структуры с массивом ДАКТ при различных значениях обратного смещения на диоде $V_{b}$ (рис. 3 ). Как следует из рис. $3, a$, при $V_{b}=0$ основное состояние электронов в КТ2 $E_{e 000}$ лежит ниже по энергии, чем в КТ1. При $V_{b}=1.1 \mathrm{~V}$ (рис. $\left.3, b\right)$ энергии основных состояний в КТ1 и КТ2 сравниваются, и становятся возможным туннелирование электронов, возбужденных в основное состояние электронов в КТ2 из состояний валентной зоны в КТ2 излучением с соответствующей длиной волны $\lambda_{m}$, в основное состояние электронов в КТ1 с последующей эмиссией (туннельной и/или термоактивированной) в матрицу GaAs, что приводит к увеличению фототока.

\section{3. Исследование спектров фоточувствительности $p-i-n$-фотодиода с ДАКТ InAs/GaAs(001)}

Измерения спектров ФЧ $p-i-n$-фотодиодов с ДАКТ InAs/GaAs(001) проводились при $300 \mathrm{~K}$ при помощи решеточного монохроматора ЛОМО МДР-2 с галогеновой лампой мощностью $100 \mathrm{~W}$ с синхронным детектированием при помощи синхронного детектора Stanford Research SR-530. Измеренные спектры фотоЭДС $V_{p h}(h v)$ нормировались на спектральное распределение интенсивности фотовозбуждения $L_{p h}(h v)$, получая, тем самым, спектр $\left.\Phi Ч S_{p h}(h v)=V_{p h}(h v) / L_{p h}(h v)\right)$.

На рис. 4 представлен спектр ФЧ $p-i-n$-фотодиода с массивом ДАКТ InAs/GaAs(001). Фотовозбуждение в спектральной области $h v<1.5 \mu \mathrm{m}$ осуществлялось через подложку $n^{+}$-GaAs. В спектре ФЧ наблюдается порог при $h v \approx 1.41 \mathrm{eV}$, связанный с краем собственной $\Phi Ч$ GaAs.

Кроме того, в спектре ФЧ наблюдаются пики в области $0.9 \mathrm{eV}<h v<1.4 \mathrm{eV}$, связанные с межзонными оптическими переходами в ДАКТ InAs. Пики с $h v_{m} \approx 1.18$ и $\approx 0.95 \mathrm{eV}$ связаны с межзонными оптическими переходами между основными состояниями дырок и электронов в КТ1 и КТ2 соответственно. Пики с $h v_{m} \approx 1.05$ и $1.3 \mathrm{eV}$ могут быть связаны с межзонными переходами между основными состояниями дырок и возбужденными электронными состояниями в КТ2 (предположительно $|200\rangle)$. Полоса ФЧ с краем при $h v 1.35 \mathrm{eV}$ связана с межзонными оптическими переходами между основными двумерными состояниями дырок и электронов смачивающего слоя (WL) InAs.

На рис. 5 приведена зависимость фототока $(300 \mathrm{~K})$ $p-i-n$-фотодиода с ДАКТ $\mathrm{InAs} / \mathrm{GaAs}(001)$ от напряжения обратного смещения $V_{b}$ при монохроматическом фотовозбуждении с $\lambda \approx 1.3 \mu \mathrm{m}$, соответствующей энергии возбуждения электрона из основного состояния в 

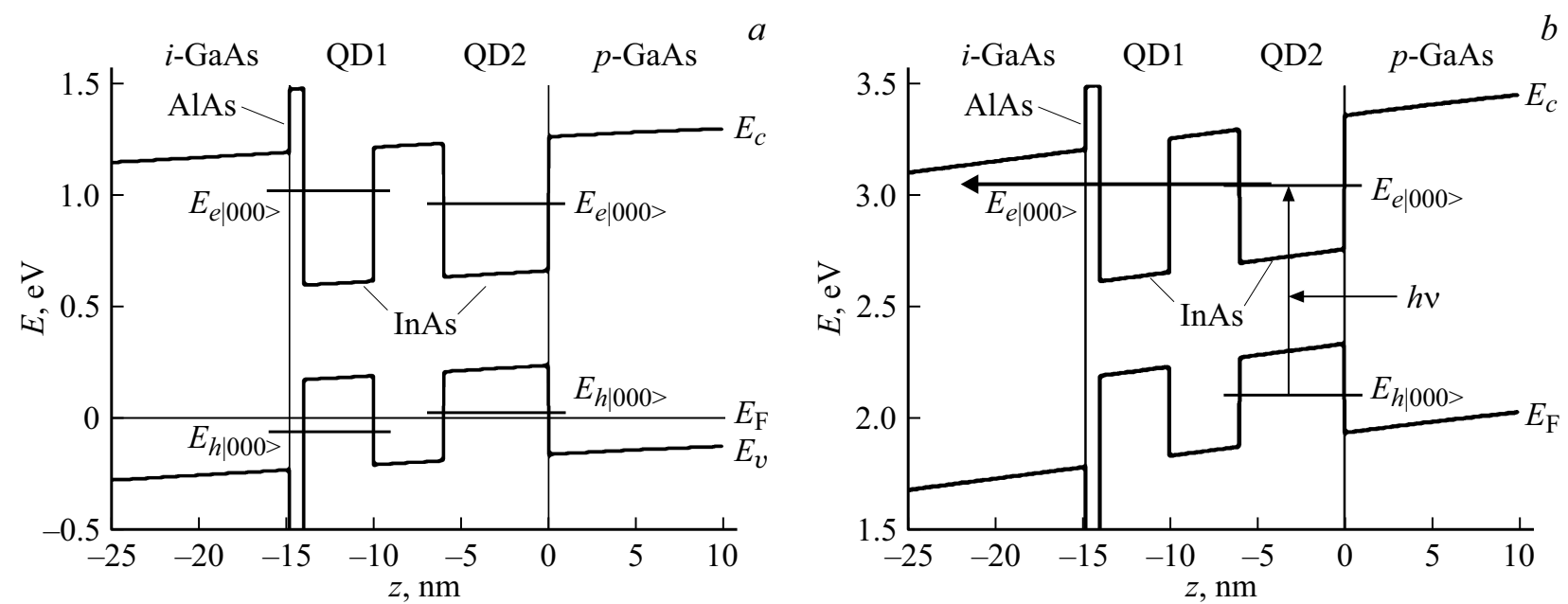

Рис. 3. Расчетные зонные диаграммы $(300 \mathrm{~K})$ ДАКТ $\mathrm{InAs} / \mathrm{GaAs}(001)$, встроенных в $p-i-n$-диод на базе GaAs. Напряжение смещения на диоде $V_{b}, \mathrm{~V}: a-0 ; b-1.1$. Стрелками показаны межзонные оптические переходы при фотовозбуждении КТ2 излучением с длиной волны $1.3 \mu \mathrm{m}$ с последующим туннелированием электрона в основное размерно-квантованное состояние электронов в КТ1 с последующей эмиссией в матрицу GaAs.

валентной зоне в основное состояние электронов в КТ2 при $300 \mathrm{~K}$. На данной зависимости наблюдаются 3 максимума, происхождение которых можно объяснить следующим образом. Механизм фотоэлектрических эффектов в гетероструктурах с КТ при межзонном фотовозбуждении КТ включает в себя в качестве обязательного и существенного элемента эмиссию фотовозбужденных носителей заряда из КТ в окружающую матрицу [15]. В связи с этим максимум 2 на рис. 5 может быть связан с возбуждением электрона из основного состояния валентной зоны на основное электронное состояние в КТ2 с последующим туннелированием электрона на основное состояние в КТ1 и эмиссией в матрицу GaAs по туннельному, термоэмиссионному или смешанному механизму (рис. $3, b$ ). Такой процесс наиболее вероятен, когда энергии основных состояний электронов в КТ1 и КТ2 одинаковы. Пики 1 и 3 на рис. 5 могут быть

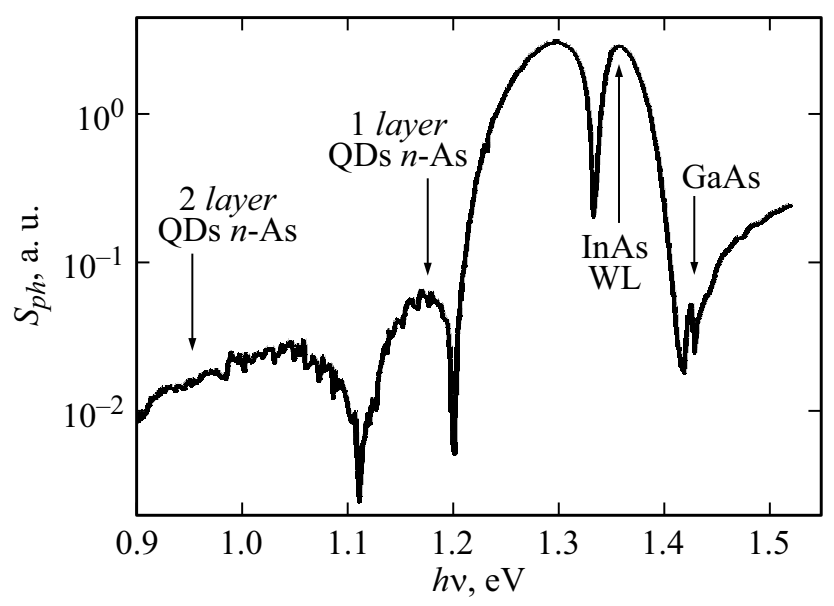

Рис. 4. Спектр ФЧ $(300 \mathrm{~K}) p-i-n$-фотодиода с ДАКТ InAs/GaAs(001).

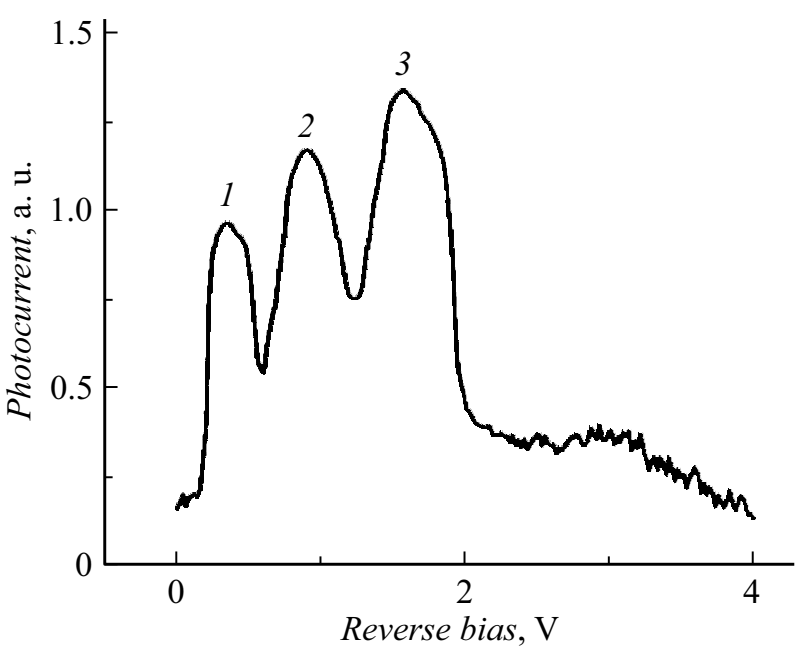

Рис. 5. Зависимость фототока $(300 \mathrm{~K}) p-i-n$-фотодиода с ДАКТ InAs/GaAs(001) от напряжения обратного смещения $V_{b}$. Длина волны фотовозбуждения $\lambda=1.3 \mu \mathrm{m} .1,3$ - туннельные межзонные оптические переходы с поглощением и испусканием оптического фонона соответственно; 2 - бесфононный межзонный переход.

связаны с туннелированием электронов из КТ2 в КТ1 с поглощением и испусканием оптических фононов соответственно.

\section{2. Особенности диссипативного туннелирования в квантовой молекуле с учетом двух фононных мод диэлектрической матрицы}

При использовании модели одномерного диссипативного туннелирования мы рассматриваем подбарьерный 


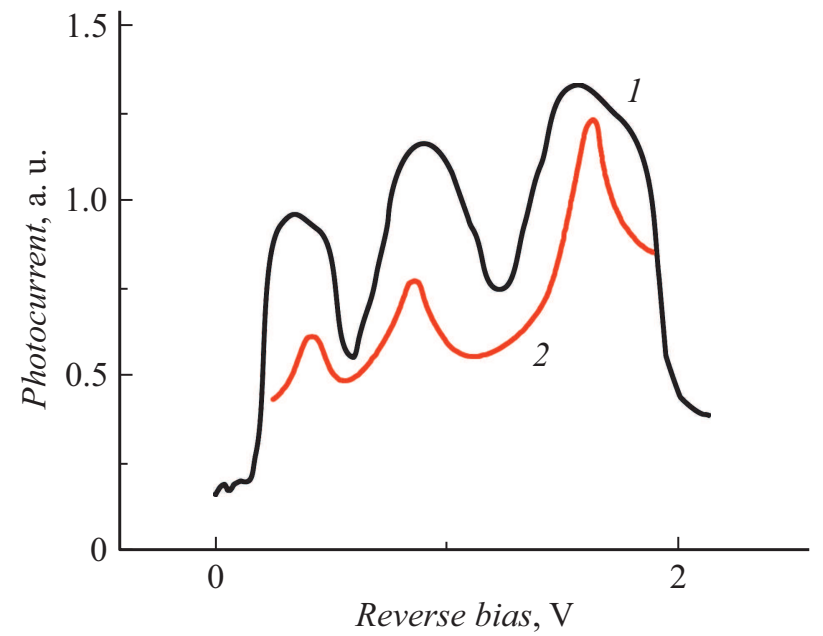

Рис. 6. Сравнение экспериментальной зависимости $S_{\mathrm{ph}}\left(V_{b}\right)$ для $p-i-n$-фотодиода с ДАКТ InAs/GaAs(001) (1) с теоретической полевой зависимостью вероятности $1 D$-диссипативного туннелирования [12] (2).

(туннельный) перенос электрона из КT2-InAs большего размера в KT1-InAs меньшего размера с учетом взаимодействия с матрицей GaAs при конечной температуре во внешнем электрическом поле. Такой процесс электронного переноса в квантовой молекуле (КМ), состоящей из двух туннельно-связанных квантовых точек (KT-InAs) разного размера, моделируется диссипативным туннелированием в двухъямном осцилляторном асимметричном потенциале вдоль координаты туннелирования. Теоретическая модель рассматривается в квазиклассическом одноинстантонном приближении, точнее приближении разреженного „газа инстантон-антиинстантонных пар“. В этом случае вероятность туннелирования удается определить аналитически с точностью до предэкспоненциального фактора $\Gamma=B \exp (-S)$ (вывод см. в Приложении), где $S$ - квазиклассическое инстантонное действие, определяемое вкладом экстремальной (инстантонной) траектории; $B$ - предэкспоненциальный фактор, определяемый вкладом пучка подбарьерных траекторий, близких к экстремальной. Соответствующий формализм был подробно описан в коллективной монографии под редакцией Нобелевского лауреата 2003 года профессора Э.Дж. Леггета с участием соавторов настоящей работы [11]. Преимущество используемой модели, как было показано в [12], состоит не только в возможности получить ответ для вероятности туннелирования в аналитическом виде, но и в случае учета влияния на процесс туннельного переноса двух локальных фононных мод, что соответствует рассматриваемому эксперименту, удается получить качественное совпадение экспериментальной туннельной ВАX для единичной КТ-InAs в системе совмещенного АCM/СТМ с теоретической полевой зависимостью вероятности $1 D$-диссипативного туннелирования [12].
В стандартной модели для определения вероятности одномерного диссипативного туннелирования в ДАКТ, мы будем использовать следующие обозначения для перенормированного двухъямного колебательного (осцилляторного) потенциала во внешнем электрическом поле: $q_{1}=b^{*}=b+\frac{|e| E}{\omega_{0}^{2}}, q_{0}=a^{*}=a-\frac{|e| E}{\omega_{0}^{2}}$, где константы $a$ и $b$ соответствуют „высотам“ КТ1-InAs и КТ2-InAs, $|e|-$ модуль заряда электрона, $E-$ напряженность электрического поля, $\omega_{0}$ - частота модельного двухъямного осцилляторного потенциала вдоль координаты туннелирования, связывающей KT1-InAs и KT2-InAs. Тогда модельный перенормированный одномерный потенциал можно представить в стандартном виде $[11,12]$. С учетом ранее полученных результатов $[11,12]$ модельный гамильтониан системы можно записать в виде

$$
\begin{aligned}
\widehat{H}= & \frac{p_{1}^{2}}{2}+V_{1}\left(y_{1}\right)+y_{1} \sum_{\alpha=2}^{N} C_{\alpha} y_{\alpha} \\
& +\frac{1}{2} \sum_{\alpha=2}^{N}\left(p_{\alpha}^{2}+\omega_{\alpha}^{2} y_{\alpha}^{2}\right),
\end{aligned}
$$

где

$$
\begin{aligned}
& V_{1}\left(y_{1}\right)=\left(\frac{1}{2} \omega_{1}^{2} y_{1}^{2}+\lambda y_{1}\right) \theta\left(-\frac{\Delta I}{2 \lambda}-y_{1}\right) \\
& +\left(\frac{1}{2} \omega_{1}^{2} y_{1}^{2}-\lambda y_{1}-\Delta I\right) \theta\left(\frac{\Delta I}{2 \lambda}+y_{1}\right) .
\end{aligned}
$$

Здесь $V_{1}\left(y_{1}\right)$ - двухъямный модельный осцилляторный потенциал, $y_{1}-$ координата туннелирования, связывающая KT1-InAs и KT2-InAs, $C_{\alpha}-$ константа электронфононного взаимодействия, $\omega_{\alpha}-$ частоты локальных фононных мод матрицы GaAs, $y_{\alpha}$ - „координаты“ локальных фононных мод, „ортонормированные“ по отношению к координате туннелирования, $\Delta I-$ параметр асимметрии двухъямного осцилляторного потенциала, линейно зависящий от напряженности внешнего электрического поля, $\theta-$ единичная функция Хевисайда, $\lambda$ - „нормировочный“ параметр, не входящий в окончательное выражение для вероятности туннелирования.

Как упоминалось выше, вероятность туннелирования частицы в единицу времени может быть найдена в квазиклассическом приближении. Для этого распад должен быть квазистационарным, т.е. ширина уровня $Г$, с которого туннелируют частицы, должна быть много меньше энергии основного состояния. Указанное условие выполняется для KT InAs/GaAs(001), составляющих квантовую молекулу [13].

Найдем одномерное квазиклассическое действие в одноинстантонном приближении с учетом влияния матрицы (среды-термостата, of the „heat-bath“):

$$
\begin{gathered}
S_{B}=2 \omega_{0}^{2}\left(q_{0}+q_{1}\right) q_{0} \tau_{0}-\frac{2 \omega_{0}^{2}\left(q_{0}+q_{1}\right)^{2} \tau_{0}^{2}}{\beta} \\
-\frac{4 \omega_{0}^{4}\left(q_{0}+q_{1}\right)^{2}}{\beta} \sum_{n=1}^{\infty} \frac{\sin ^{2} v_{n} \tau_{0}}{v_{n}^{2}\left(v_{n}^{2}+\omega_{0}^{2}+\xi_{n}\right)} .
\end{gathered}
$$


Здесь $\tau_{0}-$ „центр“ инстантона, отвечающий моменту „мнимого“ времени проскока туннелирующим электроном под верхушкой потенциального барьера модельного двухъямного осцилляторного потенциала, $\beta=\frac{\hbar \omega_{0}}{k_{B} T}-$ обезразмеренная обратная температура, $v_{n}=\frac{2 \pi n}{\beta}-$ „мацубаровская частота“, $\xi_{n}=v_{n}^{2} \sum_{\alpha=2}^{N} \frac{C_{\alpha}^{2}}{\omega_{\alpha}^{2}\left(\omega_{\alpha}^{2}+v_{n}^{2}\right)}-$ фурьекомпонента „вязкого“ ядра интегрального квазиклассического уравнения Эйлера-Лагранжа.

Предэкспоненциальный множитель определяется вкладом траекторий, близких к инстантону. Для этого мы должны разложить действие до квадратичного члена по отклонениям $q-q_{B}$ и выполнить интегрирование в функциональном пространстве. В результате вероятность туннелирования в единицу времени записывается как

$$
\begin{gathered}
\Gamma=B \exp \left(-S_{B}\right), \\
B=\left[\frac{S_{0}}{2 \pi} \frac{\operatorname{det}\left(\frac{\delta^{2} S}{\delta q^{2}}\right)_{q=-q_{0}}}{\operatorname{det}^{\prime}\left(\frac{\delta^{2} S}{\delta q^{2}}\right)_{q=q_{B}(\tau)}}\right]^{-1 / 2}, \\
S_{0}=\int_{-\beta / 2}^{\beta / 2} \dot{q}_{B}^{2}(\tau) d \tau,
\end{gathered}
$$

где $\operatorname{det}^{\prime}$ - означает, что нулевое собственное значение, соответствующее нулевой инстантонной моде, необходимо отбросить. Следует отметить, что вывод этой формулы предполагает приближение идеального инстантонного газа (точнее приближение разреженного „газа инстантон-антиинстантонных пар“)

$$
\Gamma \ll(\Delta \tau)^{-1},
$$

где $\Delta \tau-$ ширина перехода от положительного значения инстантонной траектории к отрицательному значению. Расчет предэкспоненциального множителя в рассматриваемой модели приводит к следующему ответу:

$$
\begin{aligned}
B & =\frac{2 \omega_{0}^{2}\left(q_{0}+q_{1}\right)^{2}}{(2 \pi \beta)^{1 / 2}} \sum_{n=-\infty}^{\infty} \frac{\sin ^{2} v_{n} \tau_{0}}{\lambda_{0 n}} \\
& \times\left(\sum_{n=-\infty}^{\infty} \frac{\cos 2 v_{n} \tau_{0}}{\lambda_{0 n}}\right)^{-1 / 2} .
\end{aligned}
$$

Здесь $\lambda_{0 n}=v_{n}^{2}+\omega_{0}^{2}+\xi_{n}$.

Расчет квазиклассического действия и предэкспоненциального фактора с учетом взаимодействия с двумя локальными фононными модами рассмотрен в Приложении (см. (П7), (П11)).

Проведенный аналитический расчет позволяет также учесть роль влияния локальных фононных мод среды-термостата на зависимость (см. вывод (П12)), $\Gamma=B \exp (-S)[7,11,12]$.
Полученные аналитические результаты были использованы для проведения численных оценок и сравнения с данными эксперимента. На рис. 6 показано сравнение экспериментальной зависимости $S_{\mathrm{ph}}\left(V_{b}\right)$ исследуемого фотодиода (рис. 5) с теоретической полевой зависимостью для вероятности $1 D$-диссипативного туннелирования электрона в квантовой молекуле в широкозонной матрице, рассчитанной с учетом влияния двух локальных фононных мод (TO- и LO-фононов) [7,11,12]. Максимумы на теоретической кривой обусловлены обменом энергии туннелирующего электрона с фононами [12]. Наблюдается качественное согласие теоретической и экспериментальной кривых.

\section{Заключение}

В работе получены и исследованы GaAs $p-i-n$ фотодиоды с ДАКТ InAs, исследованы их оптические и фотоэлектрические свойства. В зависимости ФЧ диодов от напряжения обратного смещения при фотовозбуждении КТ на длине волны $\approx 1.3 \mu \mathrm{m}$, соответствующей возбуждению межзонного оптического перехода между основными состояниями электронов и дырок в КТ большего размера, обнаружены 3 максимума, связанных с туннелированием фотовозбужденных электронов между ДАКТ, в том числе с поглощением и испусканием оптических фононов. Результаты эксперимента согласуются с теоретической полевой зависимостью вероятности $1 D$-диссипативного туннелирования в ДАКТ InAs/GaAs(001). Результаты работы показывают, что развитие нанотехнологии сделали возможным экспериментальное наблюдение эффектов диссипативного туннелирования электронов в искусственных размерноквантованных наноструктурах.

\section{Приложение}

Рассмотрим (3) с учетом взаимодействия с двумя локальными фононными модами $\left(\omega_{L 1}=\omega_{2}\right.$ и $\left.\omega_{L 2}=\omega_{3}\right)$. Для упрощения будем предполагать это взаимодействие достаточно малым, т.е. $\frac{C}{\omega_{0}^{2}} \ll 1$ и $\frac{C}{\omega_{L}^{2}} \ll 1$. В этом случае $\xi_{n}=v_{n}^{2} \sum_{\alpha=2}^{N} \frac{C_{\alpha}^{2}}{\omega_{\alpha}^{2}\left(\omega_{\alpha}^{2}+v_{n}^{2}\right)}$, где $v_{n}=\frac{2 \pi n}{\beta}, \beta=\frac{\hbar}{k T}$.

$$
\begin{gathered}
\xi_{n}=v_{n}^{2} \frac{C_{2}^{2}}{\omega_{2}^{2}\left(\omega_{2}^{2}+v_{n}^{2}\right)}+v_{n}^{2} \frac{C_{3}^{2}}{\omega_{3}^{2}\left(\omega_{3}^{2}+v_{n}^{2}\right)} \\
\sin ^{2} v_{n} \tau_{0}=\frac{1}{2}\left(1-\cos 2 v_{n} \tau_{0}\right) .
\end{gathered}
$$


В результате сумма в последнем слагаемом выражения (3) перепишется в виде $U=U_{1}-U_{2}$, где

$$
\begin{aligned}
& U_{1}=\frac{1}{2} \sum_{n=1}^{\infty} \frac{1}{v_{n}^{2}\left(v_{n}^{2}+\omega_{0}^{2}+v_{n}^{2} \frac{C_{2}^{2}}{\omega_{2}^{2}\left(\omega_{2}^{2}+v_{n}^{2}\right)} v_{n}^{2}+\frac{C_{3}^{2}}{\omega_{3}^{2}\left(\omega_{3}^{2}+v_{n}^{2}\right)}\right)}, \\
& U_{2}=\frac{1}{2} \sum_{n=1}^{\infty} \frac{\cos 2 v_{n} \tau_{0}}{v_{n}^{2}\left(v_{n}^{2}+\omega_{0}^{2}+v_{n}^{2} \frac{C_{2}^{2}}{\omega_{2}^{2}\left(\omega_{2}^{2}+v_{n}^{2}\right)} v_{n}^{2}+\frac{C_{3}^{2}}{\omega_{3}^{2}\left(\omega_{3}^{2}+v_{n}^{2}\right)}\right)} .
\end{aligned}
$$

Обозначим $v_{2}^{2}=x$ и преобразуем выражение в знаменателе

$$
\begin{aligned}
& x\left[\left(x+\omega_{0}^{2}\right) \omega_{2}^{2} \omega_{3}^{2}\left(x+\omega_{2}^{2}\right)\left(x+\omega_{3}^{2}\right)+x C_{2}^{2} \omega_{3}^{2}\left(x+\omega_{3}^{2}\right)\right. \\
& \left.+x C_{3}^{2} \omega_{2}^{2}\left(x+\omega_{2}^{2}\right)\right]=x\left[\omega_{2}^{4} \omega_{3}^{4} x+\omega_{2}^{2} \omega_{3}^{2} x^{3}+\omega_{3}^{2} \omega_{3}^{2} x^{2}\left(\omega_{2}^{2}+\omega_{3}^{2}\right)\right. \\
& +\omega_{2}^{4} \omega_{3}^{4} \omega_{0}^{2}+\omega_{2}^{2} \omega_{3}^{2} \omega_{0}^{2} x^{2}+\omega_{2}^{2} \omega_{3}^{2} \omega_{0}^{2} x\left(\omega_{2}^{2}+\omega_{3}^{2}\right) \\
& \left.+C_{2}^{2} \omega_{3}^{4} x+C_{2}^{2} \omega_{3}^{2} x^{2}+C_{3}^{2} \omega_{2}^{4} x+C_{3}^{2} \omega_{2}^{2} x^{2}\right] \\
& =x\left[\omega_{2}^{2} \omega_{3}^{2} x^{3}+x^{2}\left\{\omega_{2}^{2} \omega_{3}^{2}\left(\omega_{2}^{2}+\omega_{3}^{2}\right)+\omega_{0}^{2} \omega_{2}^{2} \omega_{3}^{2}+C_{2}^{2} \omega_{3}^{2}\right.\right. \\
& \left.+C_{3}^{2} \omega_{2}^{2}\right\}+x\left\{\omega_{2}^{4} \omega_{3}^{4}+\omega_{0}^{2} \omega_{2}^{2} \omega_{3}^{2}\left(\omega_{2}^{2}+\omega_{3}^{2}\right)\right. \\
& \left.\left.+C_{2}^{2} \omega_{3}^{4}+C_{3}^{2} \omega_{2}^{4}\right\}+\omega_{0}^{2} \omega_{2}^{4} \omega_{3}^{4}\right] \\
& =x \omega_{2}^{2} \omega_{3}^{2}\left[x^{3}+x^{2}\left\{\omega_{2}^{2}+\omega_{3}^{2}+\omega_{0}^{2}+\frac{C_{2}^{2}}{\omega_{2}^{2}}+\frac{C_{3}^{2}}{\omega_{3}^{2}}\right\}\right. \\
& \left.+x\left\{\omega_{2}^{2} \omega_{3}^{3}+\omega_{0}^{2}\left(\omega_{2}^{2}+\omega_{3}^{2}\right)+\frac{C_{2}^{2} \omega_{3}^{2}}{\omega_{2}^{2}}+\frac{C_{3}^{2} \omega_{2}^{2}}{\omega_{3}^{2}}\right\}+\omega_{0}^{2} \omega_{2}^{2} \omega_{3}^{2}\right]
\end{aligned}
$$

Введем обозначения $A=\omega_{2}^{2}+\omega_{3}^{2}+\omega_{0}^{2}+\frac{C_{2}^{2}}{\omega_{2}^{2}}+\frac{C_{3}^{2}}{\omega_{3}^{2}}$,

$$
\begin{gathered}
B_{\omega}=\omega_{2}^{2} \omega_{3}^{2}+\omega_{0}^{2}\left(\omega_{2}^{2}+\omega_{3}^{2}\right)+\frac{C_{2}^{2} \omega_{3}^{2}}{\omega_{2}^{2}}+\frac{C_{3}^{2} \omega_{2}^{2}}{\omega_{3}^{2}}, \\
C=\omega_{0}^{2} \omega_{2}^{2} \omega_{3}^{2} .
\end{gathered}
$$

Тогда выражение в знаменателе первого слагаемого в (П1) примет вид

$$
\begin{aligned}
& x \omega_{2}^{2} \omega_{3}^{2}[\underbrace{x^{3}+A x^{2}+B_{\omega} x+C}_{=0}] \\
& =x \omega_{2}^{2} \omega_{3}^{2}\left(x-x_{1}\right)\left(x-x_{2}\right)\left(x-x_{3}\right) .
\end{aligned}
$$

Обозначим $\quad Q=\frac{A^{2}-3 B_{\omega}}{9} ; \quad R=\frac{2 A^{3}-9 A B_{\omega}+27 C}{54}$; $S=Q^{3}-R^{2} ; \Phi=\frac{1}{3} \arccos \left(\frac{R}{\sqrt{Q^{3}}}\right)$. Если $S>0$, тогда

$$
\begin{gathered}
x_{1}=-2 \sqrt{Q} \cos (\Phi)-\frac{A}{3}, \\
x_{2}=-2 \sqrt{Q} \cos \left(\Phi+\frac{2}{3} \pi\right)-\frac{A}{3}, \\
x_{3}=-2 \sqrt{Q} \cos \left(\Phi-\frac{2}{3} \pi\right)-\frac{A}{3} .
\end{gathered}
$$

Первая сумма в (П1) преобразуется к виду

$$
U_{1}=\frac{1}{2} \sum_{n=1}^{\infty} \frac{\omega_{2}^{2} \omega_{3}^{2}\left(\omega_{2}^{2}+v_{n}^{2}\right)\left(\omega_{3}^{2}+v_{n}^{2}\right)}{v_{n}^{2} \omega_{2}^{2} \omega_{3}^{2}\left(v_{n}^{2}-x_{1}\right)\left(v_{n}^{2}-x_{2}\right)\left(v_{n}^{2}-x_{3}\right)} .
$$

Последнее выражение (П1) разобъем на простые дроби

$$
\begin{aligned}
\frac{\beta_{0}}{x} & +\frac{\gamma}{x-x_{1}}+\frac{\varphi}{x-x_{2}}+\frac{\Delta}{x-x_{3}} \\
& =\frac{x^{2}+x\left(\omega_{2}^{2}+\omega_{3}^{2}\right)+\omega_{2}^{2} \omega_{3}^{2}}{x\left(x-x_{1}\right)\left(x-x_{2}\right)\left(x-x_{3}\right)},
\end{aligned}
$$

где

$$
\beta_{0}=-\frac{\omega_{2}^{2} \omega_{3}^{2}}{x_{1} x_{2} x_{3}}
$$

$$
\begin{aligned}
& \Delta=\frac{x_{3}^{2}}{\left(x_{3}-x_{2}\right)\left(x_{1}-x_{3}\right)}\left\{\frac{\omega_{2}^{2} \omega_{3}^{2}}{x_{1} x_{2} x_{3}}\left(\frac{x_{1} x_{2}+x_{1} x_{3}+x_{2} x_{3}}{x_{2} x_{3}}-1\right)\right. \\
&+ \frac{\omega_{2}^{2}+\omega_{3}^{2}}{x_{2} x_{3}}-\frac{1}{x_{3}}\left(1+\frac{\omega_{2}^{2} \omega_{3}^{2}}{x_{1} x_{2} x_{3}}\left[\frac{x_{1} x_{2}+x_{1} x_{3}+x_{2} x_{3}}{x_{2} x_{3}}\right.\right. \\
&+\left.\left.\left.\left(x_{2}+x_{3}-x_{1}\right)\right]\right)+\frac{\left(\omega_{2}^{2}+\omega_{3}^{2}\right)\left(x_{2}+x_{3}\right)}{x_{2} x_{3}}\right\}, \\
& \varphi=\frac{x_{2}}{x_{3}\left(x_{2}-x_{1}\right)}\left\{\Delta \frac{x_{2}}{x_{3}}\left(x_{1}-x_{3}\right)-1\right. \\
&-\frac{\omega_{2}^{2} \omega_{3}^{2}}{x_{1} x_{2} x_{3}}\left(x_{2}+x_{3}-x_{1}\right)-\frac{x_{2}+x_{3}}{x_{2} x_{3}} \\
&\left.\times\left\{\omega_{2}^{2}+\omega_{3}^{2}+\frac{\omega_{2}^{2} \omega_{3}^{2}}{x_{1} x_{2} x_{3}}\left(x_{1} x_{2}+x_{1} x_{3}+x_{2} x_{3}\right)\right\}\right\} . \\
& \gamma=\frac{1}{x_{2} x_{3}}\left\{\omega_{2}^{2}+\omega_{3}^{2}-\Delta x_{1} x_{2}-\varphi_{1} x_{3}\right. \\
&\left.\quad-\beta_{0}\left(x_{2} x_{3}+x_{1}\left(x_{2}+x_{3}\right)\right)\right\} \quad v_{n}=\frac{2 \pi n}{\beta} .
\end{aligned}
$$

В итоге $U_{1}$ преобразуется к виду

$$
\begin{gathered}
U_{1}=\frac{1}{2} \sum_{n=1}^{\infty}\left(\frac{\beta_{0}}{v_{n}^{2}}+\frac{\gamma}{v_{n}^{2}-x_{1}}+\frac{\varphi}{v_{n}^{2}-x_{2}}+\frac{\Delta}{v_{n}^{2}-x_{3}}\right) . \\
\sum_{n=1}^{\infty} \frac{\beta_{0}}{v_{n}^{2}}=\beta_{0} \sum_{n=1}^{\infty} \frac{\beta^{2}}{4 \pi^{2} n^{2}}=\beta_{0} \frac{\beta^{2}}{4 \pi^{2}} \sum_{n=1}^{\infty} \frac{1}{n^{2}}=\beta_{0} \frac{\beta^{2}}{24}
\end{gathered}
$$

$x_{1}=-2 \sqrt{Q} \cos \phi-\frac{A}{3}=-x_{10}=-\left(2 \sqrt{Q} \cos \phi+\frac{A}{3}\right)$;

Если $x_{1}<0$;

$$
\begin{aligned}
& \sum_{n=1}^{\infty} \frac{\gamma}{v_{n}^{2}+x_{10}}=\sum_{n=1}^{\infty} \frac{\gamma}{\frac{4 \pi^{2} n^{2}}{\beta^{2}}+x_{10}}=\frac{\gamma \beta^{2}}{4 \pi^{2}} \sum_{n=1}^{\infty} \frac{1}{n^{2}+\frac{x_{10} \beta^{2}}{4 \pi^{2}}} \\
& =\frac{\gamma \beta^{2}}{4 \pi^{2}}\left[-\frac{4 \pi^{2}}{2 x_{1} \beta^{2}}-\frac{\pi^{2}}{\sqrt{x_{1}} \beta} \operatorname{ctg}\left(\frac{\sqrt{x_{1}} \beta}{2 \pi}\right)\right] ; \tilde{x}_{10}^{2}=\frac{x_{10} \beta^{2}}{4 \pi^{2}}, \\
& x_{2}=-2 \sqrt{Q} \cos \left(\Phi+\frac{2}{3} \pi\right)-\frac{A}{3}=-x_{20} \quad \tilde{x}_{20}^{2}=\frac{x_{20} \beta^{2}}{4 \pi^{2}}
\end{aligned}
$$


$x_{3}=-2 \sqrt{Q} \cos \left(\Phi-\frac{2}{3} \pi\right)-\frac{A}{3}=-x_{30} \quad \tilde{x}_{30}^{2}=\frac{x_{30} \beta^{2}}{4 \pi^{2}}$.

Если $x_{1}>0, x_{2}>0, x_{3}>0$ :

$$
\begin{aligned}
& U_{1}=\frac{1}{2}\left\{\beta_{0} \frac{\beta^{2}}{24}+\frac{\gamma \beta^{2}}{4 \pi^{2}}\left[-\frac{4 \pi^{2}}{2 x_{1} \beta^{2}}-\frac{\pi^{2}}{\sqrt{x_{1}} \beta} \operatorname{ctg}\left(\frac{\sqrt{x_{1}} \beta}{2}\right)\right]\right. \\
& +\frac{\varphi \beta^{2}}{4 \pi^{2}}\left[-\frac{4 \pi^{2}}{2 x_{2} \beta^{2}}-\frac{\pi^{2}}{\sqrt{x_{2}} \beta} \operatorname{ctg}\left(\frac{\sqrt{x_{2}} \beta}{2}\right)\right] \\
& \left.+\frac{\Delta \beta^{2}}{4 \pi^{2}}\left[-\frac{4 \pi^{2}}{2 x_{3} \beta^{2}}-\frac{\pi^{2}}{\sqrt{x_{3}} \beta} \operatorname{ctg}\left(\frac{\sqrt{x_{3}} \beta}{2}\right)\right]\right\} .
\end{aligned}
$$

Перейдем к вычислению $U_{2}$ :

$$
\begin{aligned}
& U_{2}=\frac{1}{2} \sum_{n=1}^{\infty}\left(\frac{\beta_{0} \cos 2 v_{n} T_{0}}{v_{n}^{2}}+\frac{\gamma \cos 2 v_{n} T_{0}}{v_{n}^{2}-x_{1}}\right. \\
& \left.+\frac{\varphi \cos 2 v_{n} T_{0}}{v_{n}^{2}-x_{2}}+\frac{\Delta \cos 2 v_{n} T_{0}}{v_{n}^{2}-x_{3}}\right), \\
& \frac{1}{2} \sum_{n=1}^{\infty} \frac{\beta_{0} \cos ^{2} \frac{2 \pi T_{0} n}{\beta}}{\frac{4 \pi^{2} n^{2}}{\beta^{2}}}=\frac{1}{2}\left[\frac{\beta^{2} \beta_{0}}{4 \pi^{2}} \sum_{n=1}^{\infty} \frac{\cos ^{2} \frac{2 \pi T_{0}}{\beta} n}{n^{2}}\right] \\
& =\frac{1}{2}\left[\frac{\beta^{2} \beta_{0}}{4 \pi^{2}} \frac{1}{12}\left(3 \frac{\left(4 \pi T_{0}\right)^{2}}{\beta}-6 \pi \frac{4 \pi T_{0}}{\beta}+2 \pi^{2}\right)\right] \\
& +\frac{1}{2} \gamma \sum_{n=1}^{\infty} \frac{\cos \frac{4 \pi T_{0} n}{\beta}}{\frac{4 \pi^{2} n^{2}}{\beta^{2}}-x_{1}}=\frac{1}{2}\left[\frac{\beta^{2} \gamma}{4 \pi^{2}} \sum_{n=1}^{\infty} \frac{\cos \frac{4 \pi T_{0}}{\beta} n}{n^{2}-\frac{x_{1} \beta^{2}}{4 \pi^{2}}}\right] \\
& =\frac{1}{2}\left[\frac { \beta ^ { 2 } \gamma } { 4 \pi ^ { 2 } } \left\{\frac{\pi^{2}}{\sqrt{x_{1}} \beta} \cos \left[\left(\pi-\frac{4 \pi T_{0}}{\beta}\right) \frac{\sqrt{x_{1}} \beta}{2 \pi}\right]\right.\right. \\
& \left.\left.\times \operatorname{cosec} \frac{\sqrt{x_{1}} \beta}{2}+\frac{2 \pi^{2}}{x_{1} \beta^{2}}\right\}\right] .
\end{aligned}
$$

При $x_{1}, x_{2}, x_{3}>0$

$$
\begin{aligned}
& U_{2}=\frac{1}{2}\left\{\frac{\beta_{0} \beta^{2}}{48}\left(3 \frac{\left(4 \pi T_{0}\right)^{2}}{\beta}-\frac{24 \pi^{2} T_{0}}{\beta}+2 \pi^{2}\right)\right. \\
& +\frac{\gamma \beta^{2}}{4 \pi^{2}}\left\{\frac{\pi^{2}}{\sqrt{x_{1}} \beta} \cos \left[\left(\pi-\frac{4 \pi T_{0}}{\beta}\right) \frac{\sqrt{x_{1}} \beta}{2 \pi}\right] \operatorname{cosec} \frac{\sqrt{x_{1}} \beta}{2}\right\} \\
& +\frac{\varphi \beta^{2}}{4 \pi^{2}}\left\{\frac{\pi^{2}}{\sqrt{x_{2}} \beta} \cos \left[\left(\pi-\frac{4 \pi T_{0}}{\beta}\right) \frac{\sqrt{x_{2}} \beta}{2 \pi}\right]\right. \\
& \left.\times \operatorname{cosec} \frac{\sqrt{x_{2}} \beta}{2}-\frac{2 \pi^{2}}{x_{2} \beta^{2}}\right\}+\frac{\Delta \beta^{2}}{4 \pi^{2}}\left\{\frac { \pi ^ { 2 } } { \sqrt { x _ { 3 } } \beta } \operatorname { c o s } \left[\left(\pi-\frac{4 \pi T_{0}}{\beta}\right)\right.\right. \\
& \left.\left.\left.\times \frac{\sqrt{x_{3}} \beta}{2 \pi}\right] \operatorname{cosec} \frac{\sqrt{x_{3}} \beta}{2}-\frac{2 \pi^{2}}{x_{3} \beta^{2}}\right\}\right\} .
\end{aligned}
$$

Квазиклассическое действие с учетом двух „промотирующих“ локальных фононных мод сводится к выражению вида

$$
\begin{gathered}
S_{B} 2 \omega_{0}^{2}(a+b) a \tau_{0}-\frac{2}{\beta} \omega_{0}^{2}(a+b)^{2} \tau_{0}^{2} \\
-\frac{4}{\beta} \omega_{0}^{4}(a+b)^{2}\left\{U_{1}+U_{2}\right\},
\end{gathered}
$$

где

$$
\begin{aligned}
\tau_{0} & =\frac{1}{2 \omega_{0}} \operatorname{Arcsh}\left[\frac{b-a}{b+a} \operatorname{sh} \frac{\omega_{0} \beta}{4}\right]+\frac{\beta}{4} \\
& =\frac{1}{2 \omega_{0}} \operatorname{Arcsh}\left[\frac{\frac{b}{a}-1}{\frac{b}{a}+1} \operatorname{sh} \frac{\omega_{0} \beta}{4}\right]+\frac{\beta}{4}
\end{aligned}
$$

или

$$
\begin{aligned}
\tau_{0}^{*}=\tau_{0} \omega_{0} & =\frac{1}{2} \operatorname{Arcsh}\left[\frac{b^{*}-1}{b^{*}+1} \operatorname{sh} \beta^{*}\right]+\beta^{*} ; \\
\tau_{0}^{*} & =\tau \omega_{0} ; \quad \beta^{*}=\frac{\omega_{0} \beta}{4} .
\end{aligned}
$$

Окончательно перенормированное выражение для одномерного квазиклассического инстантонного действия с учетом двух локальных фононных мод среды-термостата принимает вид

$$
\begin{aligned}
& \tilde{S}_{10}=\frac{S_{10}}{\omega_{0} a^{2}}=2\left(b^{*}+1\right) \tau_{0}^{*}-\frac{1}{2 \beta^{*}}\left(b^{*}+1\right)^{2} \tau_{0}^{* 2}-\frac{\left(b^{*}+1\right)^{2}}{\beta^{*}} \\
& \times\left\{\frac { 1 } { 2 } \left[\beta_{0} \omega_{0}^{2}\left(\frac{\beta \omega_{0}}{4}\right)^{2} \frac{2}{3}+4 \frac{\gamma \omega_{0}^{2}\left(\frac{\beta \omega_{0}}{4}\right)^{2}}{\pi^{2}}\right.\right. \\
& \times\left[-\frac{4 \pi^{2}}{2 x_{1} \beta^{2}}-\frac{\pi^{2}}{\sqrt{x_{1}} \beta} \operatorname{ctg}\left(\frac{\sqrt{x_{1} \beta}}{2 \pi}\right)\right]+4 \frac{\varphi \omega_{0}^{2} \beta^{* 2}}{\pi^{2}} \\
& \times\left[-\frac{4 \pi^{2}}{2 x_{2} \beta^{2}}-\frac{\pi^{2}}{\sqrt{x_{2}} \beta} \operatorname{ctg}\left(\frac{\sqrt{x_{2}} \beta}{2 \pi}\right)\right] \\
& \left.+4 \frac{\Delta \omega_{0}^{2} \beta^{* 2}}{\pi^{2}}\left[-\frac{4 \pi^{2}}{2 x_{3} \beta^{2}}-\frac{\pi^{2}}{\sqrt{x_{3}} \beta} \operatorname{ctg}\left(\frac{\sqrt{x_{3} \beta}}{2 \pi}\right)\right]\right] \\
& -\frac{1}{2}\left[\beta_{0} \omega_{0}^{2}\left(\frac{\beta \omega_{0}}{4}\right)^{2} \frac{1}{3}\left(3\left(\frac{4 \pi \tau_{0} \omega_{0}}{\beta \omega_{0}}\right){ }^{2}-\frac{6 \pi^{2} \tau_{0} \omega_{0} 4}{\beta \omega_{0}}+2 \pi^{2}\right)\right. \\
& +\frac{4 \gamma \omega_{0}^{2}\left(\frac{\beta \omega_{0}}{4}\right)^{2}}{\pi^{2}}\left\{\frac{\omega_{0} \pi^{2} 4}{4 \sqrt{x_{1}} \beta \omega_{0}} \cos \left[\left(\pi-\frac{4 \pi \tau_{0} \omega_{0}}{\beta \omega_{0}}\right) \frac{\sqrt{x_{1}} 2 \beta \omega_{0}}{\omega_{0} \pi 4}\right]\right. \\
& \left.\times \operatorname{cosec} \frac{2 \sqrt{x 1}}{\omega_{0}} \frac{\beta_{0} \omega_{0}}{4}+\frac{\omega_{0}^{2} \pi^{2} 4}{8 x_{1} \beta \omega_{0}}\right\}+\frac{4 \phi \omega_{0}^{2} \beta^{* 2}}{\pi^{2}} \\
& \times\left\{\frac{\omega_{0} \pi^{2} 4}{4 \sqrt{x_{2}} \beta^{*}} \cos \left[\left(\pi-\frac{4 \pi \tau_{0}^{*} \omega_{0}}{\beta^{*}}\right) \frac{\sqrt{x_{2}} 2 \beta^{*}}{\omega_{0} \pi}\right]\right. \\
& \left.\times \operatorname{cosec} \frac{2 \sqrt{x_{2}}}{\omega_{0}} \beta^{*}+\frac{\omega_{0}^{2} \pi^{2}}{8 x_{20} \beta^{* 2}}\right\}+\frac{4 \Delta \omega_{0}^{2} \beta^{* 2}}{\pi^{2}} \\
& \times\left\{\frac{\omega_{0} \pi^{2} 4}{4 \sqrt{x_{3}} \beta^{*}} \cos \left[\left(\pi-\frac{4 \pi \tau_{0}^{*} \omega_{0}}{\beta^{*}}\right) \frac{\sqrt{x_{3}} 2 \beta^{*}}{\omega_{0} \pi} \beta^{*}+\frac{\omega_{0}^{2} \pi^{2}}{8 x_{3} \beta^{* 2}}\right\}\right] . \\
& \times \operatorname{cosec} \\
& \times
\end{aligned}
$$

Перейдем к вычислению предэкспоненциального фактора с учетом двух промотирующих локальных фонон- 
ных мод:

$$
B=\frac{2 \omega_{0}^{2}(a+b)^{2}}{(2 \pi \beta)^{\frac{1}{2}}} \frac{\sum_{n=-\infty}^{\infty} \frac{\sin ^{2} v_{n} \tau_{0}}{\lambda_{0 n}}}{\left[\sum_{n=-\infty}^{\infty} \frac{\cos 2 v_{n} \tau_{0}}{\lambda_{0 n}}\right]^{\frac{1}{2}}},
$$

где

$$
\begin{gathered}
\lambda_{0 n}=v_{n}^{2}+\omega_{0}^{2}+\xi_{n}, \\
\sum_{-\infty}^{\infty} \frac{\sin ^{2} v_{n} \tau_{0}=\frac{1}{2}\left(1-\cos 2 v_{n} \tau_{0}\right)}{v_{n}^{2}+\omega_{0}^{2}+\frac{v_{n}^{2} C_{2}^{2}}{\omega_{2}^{2}\left(\omega_{2}^{2}+v_{n}^{2}\right)}+\frac{v_{n}^{2} C_{3}^{2}}{\omega_{3}^{2}\left(\omega_{3}^{2}+v_{n}^{2}\right)}} \\
=\frac{1}{2} \sum_{n=-\infty}^{\infty} \frac{\left(1-\cos 2 v_{n} \tau_{0}\right) \omega_{2}^{2} \omega_{3}^{2}\left(\omega_{2}^{2}+v_{n}^{2}\right)\left(\omega_{3}^{2}+v_{n}^{2}\right)}{\left(\omega_{0}^{2}+v_{n}^{2}\right) \omega_{2}^{2} \omega_{3}^{2}\left(\omega_{2}^{2}+v_{n}^{2}\right)\left(\omega_{3}^{2}+v_{n}^{2}\right)+}+v_{n}^{2} C_{2}^{2} \omega_{3}^{2}\left(\omega_{3}^{2}+v_{n}^{2}\right)+v_{2}^{2} C_{3}^{2} \omega_{3}^{2}\left(\omega_{2}^{2}+v_{n}^{2}\right) \\
x=v_{n}^{2}
\end{gathered}
$$

где введены обозначения $A=\omega_{2}^{2}+\omega_{3}^{2}+\omega_{0}^{2}+\frac{C_{2}^{2}}{\omega_{2}^{2}}+\frac{C_{3}^{2}}{\omega_{3}^{2}}$,

$$
B_{\omega}=\omega_{2}^{2} \omega_{3}^{2}+\omega_{0}^{2}\left(\omega_{2}^{2}+\omega_{3}^{2}\right) \frac{C_{2}^{2} \omega_{3}^{2}}{\omega_{2}^{2}}+\frac{C_{3}^{2} \omega_{2}^{2}}{\omega_{3}^{2}}
$$$$
C=\omega_{0}^{2} \omega_{2}^{2} \omega_{3}^{2} \text {, }
$$

обозначим также

$$
\begin{gathered}
Q=\frac{A^{2}-3 B_{\omega}}{9} ; \quad R=\frac{2 A^{3}-9 A B_{\omega}+27 C}{54} \\
S=Q^{3}-R^{2} ;
\end{gathered}
$$

При $S>0$

$$
\begin{gathered}
x_{1}=-2 \sqrt{Q} \cos (\Phi)-\frac{A}{3}, \\
x_{2}=-2 \sqrt{Q} \cos \left(\Phi+\frac{2}{3} \pi\right)-\frac{A}{3}, \\
x_{3}=-2 \sqrt{Q} \cos \left(\Phi-\frac{2}{3} \pi\right)-\frac{A}{3} .
\end{gathered}
$$

Разложим знаменатель соотношения (П9)

$$
\begin{gathered}
=\frac{1}{2} \sum_{n=-\infty}^{\infty} \frac{\left(\omega_{2}^{2}+v_{n}^{2}\right)\left(\omega_{3}^{2}+v_{n}^{2}\right)}{\left(v_{n}^{2}-x_{1}\right)\left(v_{n}^{2}-x_{2}\right)\left(v_{n}^{2}-x_{3}\right)} \\
=\frac{D}{v_{n}^{2}-x_{1}}+\frac{E}{v_{n}^{2}-x_{2}}+\frac{F}{v_{n}^{2}-x_{3}} \\
F=\frac{\left(\omega_{2}^{2}+\omega_{3}^{2}+x_{2}+x_{3}\right)\left[x_{2} x_{3}\left(x_{1}+x_{3}\right)-x_{1} x_{3}\left(x_{2}+x_{3}\right)\right]+}{\left.\left(x_{2}-x_{1}\right)\left[x_{1} x_{2}\left(x_{1}\right)\left[x_{3}\right)-x_{2}\right) \omega_{2}^{2} \omega_{3}^{2}+x_{2} x_{3}\left(\omega_{2}^{2}+\omega_{3}^{2}\right)\right]} \\
E=\frac{\left.\left.\omega_{2}^{2}+x_{2}\right)\right]-}{-\omega_{3}^{2}+x_{3}\left[x_{2} x_{3}\left(x_{1}+x_{3}\right)-x_{1} x_{3}\left(x_{2}+x_{3}\right)\right]} \\
x_{2}-x_{1}+F\left(x_{1}-x_{3}\right)
\end{gathered}
$$

$$
D=-\frac{\omega_{2}^{2}+\omega_{3}^{2}+E\left(x_{1}+x_{3}\right)+F\left(x_{1}+x_{2}\right)}{x_{2}+x_{3}}
$$$$
\frac{1}{2} \sum_{n=-\infty}^{\infty} \frac{D}{v_{n}^{2}-x_{1}}=\frac{D}{2} \sum_{n=-\infty}^{\infty} \frac{1}{\frac{4 \pi^{2} n^{2}}{\beta^{2}}-x_{1}}
$$$$
=\frac{1}{2} \frac{D \beta^{2}}{4 \pi^{2}} \sum_{n=-\infty}^{\infty} \frac{1}{n^{2}-\frac{x_{1} \beta^{2}}{4 \pi^{2}}}=\frac{1}{2} \frac{D \beta^{2}}{4 \pi^{2}}
$$$$
\left[-\frac{4 \pi^{2}}{x_{1} \beta^{2}}+2 \sum_{n=-\infty}^{\infty} \frac{1}{n^{2}-\frac{x_{1} \beta^{2}}{4 \pi^{2}}}\right]
$$

(при $\left.x_{1}>0\right)$ :

$=\frac{1}{2} \frac{D \beta^{2}}{4 \pi^{2}}\left[-\frac{4 \pi^{2}}{x_{1} \beta^{2}}+2\left\{-\frac{2 \pi^{2}}{x_{10} \beta^{2}}-\frac{\pi^{2}}{\sqrt{x_{1}} \beta} \operatorname{ctg} \frac{\sqrt{x_{1} \beta}}{2}\right\}\right]$.

Сумма, содержащая $\cos 2 v_{n} \tau_{0}$, дает в этом случае

$$
\begin{aligned}
= & -\frac{1}{2} \frac{D \beta^{2}}{4 \pi^{2}}\left[-\frac{4 \pi^{2}}{x_{1} \beta^{2}}+2\left\{-\frac{\pi^{2}}{\sqrt{x_{1}} \beta} \cos \left[\left(\pi-\frac{4 \pi \tau_{0}}{\beta}\right)\right.\right.\right. \\
& \left.\left.\left.\times \frac{\sqrt{x_{1}} \beta}{2 \pi}\right] \operatorname{cosec} \frac{\sqrt{x_{1}} \beta}{2}+\frac{2 \pi^{2}}{x_{1} \beta}\right\}\right] .
\end{aligned}
$$

В итоге обезразмеренный предэкспоненциальный фактор определяется суммами двух типов:

$$
\tilde{B}=\frac{B}{a^{2} \omega^{\frac{2}{3}}}=\frac{2 \omega_{0}^{2}\left(\frac{b}{a}+1\right)^{2}}{(2 \pi \beta)^{\frac{1}{2}}} \frac{V_{1}}{\left(V_{2}\right)^{\frac{1}{2}}},
$$

$$
\begin{aligned}
& V_{1}=\sum_{n=-\infty}^{\infty} \frac{\sin ^{2} v_{n} \tau_{0}}{\lambda_{0 n}}=\frac{1}{2} \frac{D \beta^{2}}{4 \pi^{2}}\left[-\frac{4 \pi^{2}}{x_{1} \beta^{2}}+2\left\{-\frac{2 \pi^{2}}{x_{10} \beta^{2}}\right.\right. \\
& \left.\left.-\frac{\pi^{2}}{\sqrt{x_{1}} \beta} \operatorname{ctg} \frac{\sqrt{x_{1} \beta}}{2}\right\}\right]+\frac{1}{2} \frac{E \beta^{2}}{4 \pi^{2}}\left[-\frac{4 \pi^{2}}{x_{2} \beta^{2}}+2\left\{-\frac{2 \pi^{2}}{x_{20} \beta^{2}}\right.\right. \\
& \left.\left.-\frac{\pi^{2}}{\sqrt{x_{2}} \beta} \operatorname{ctg} \frac{\sqrt{x_{2} \beta}}{2}\right\}\right]+\frac{1}{2} \frac{F \beta^{2}}{4 \pi^{2}}\left[-\frac{4 \pi^{2}}{x_{3} \beta^{2}}+2\left\{-\frac{2 \pi^{2}}{x_{30} \beta^{2}}\right.\right. \\
& \left.\left.-\frac{\pi^{2}}{\sqrt{x_{3}} \beta} \operatorname{ctg} \frac{\sqrt{x_{3} \beta}}{2}\right\}\right]-\frac{1}{2} \frac{D \beta^{2}}{4 \pi^{2}}\left[-\frac{4 \pi^{2}}{x_{1} \beta^{2}}+2\left\{-\frac{\pi^{2}}{\sqrt{x_{1} \beta}}\right.\right. \\
& \left.\left.\times \cos \left[\left(\pi-\frac{4 \pi \tau_{0}}{\beta}\right) \frac{\sqrt{x_{1}} \beta}{2 \pi}\right] \operatorname{cosec} \frac{\sqrt{x_{1} \beta}}{2}+\frac{2 \pi^{2}}{x_{1} \beta^{2}}\right\}\right] \\
& -\frac{1}{2} \frac{E \beta^{2}}{4 \pi^{2}}\left[-\frac{4 \pi^{2}}{x_{2} \beta^{2}}+2\left\{-\frac{\pi^{2}}{\sqrt{x_{2}} \beta} \cos \left[\left(\pi-\frac{4 \pi \tau_{0}}{\beta}\right) \frac{\sqrt{x_{2}} \beta}{2 \pi}\right]\right.\right. \\
& \left.\left.\times \operatorname{cosec} \frac{\sqrt{x_{2} \beta}}{2}+\frac{2 \pi^{2}}{x_{2} \beta^{2}}\right\}\right]-\frac{1}{2} \frac{F \beta^{2}}{4 \pi^{2}}\left[-\frac{4 \pi^{2}}{x_{3} \beta^{2}}+2\left\{-\frac{\pi^{2}}{\sqrt{x_{3}} \beta}\right.\right. \\
& \left.\left.\times \cos \left[\left(\pi-\frac{4 \pi \tau_{0}}{\beta}\right) \frac{\sqrt{x_{3}} \beta}{2 \pi}\right] \operatorname{cosec} \frac{\sqrt{x_{3} \beta}}{2}+\frac{2 \pi^{2}}{x_{3} \beta^{2}}\right\}\right] .
\end{aligned}
$$




$$
\begin{aligned}
& V_{2}=\sum_{n=-\infty}^{\infty} \frac{\cos 2 v_{n} \tau_{0}}{\lambda_{0 n}}=\frac{D \beta^{2}}{4 \pi^{2}}\left[-\frac{4 \pi^{2}}{x_{1} \beta^{2}}\right. \\
& +2\left\{-\frac{\pi^{2}}{\sqrt{x_{1}} \beta} \cos \left[\left(\pi-\frac{4 \pi \tau_{0}}{\beta}\right)\right.\right. \\
& \left.\left.\left.\times \frac{\sqrt{x_{1}} \beta}{2 \pi}\right] \operatorname{cosec} \frac{\sqrt{x_{1}} \beta}{2}+\frac{2 \pi^{2}}{x_{1} \beta^{2}}\right\}\right]+\frac{E \beta^{2}}{4 \pi^{2}}\left[-\frac{4 \pi^{2}}{x_{2} \beta^{2}}\right. \\
& +2\left\{-\frac{\pi^{2}}{\sqrt{x_{2}} \beta} \cos \left[\left(\pi-\frac{4 \pi \tau_{0}}{\beta}\right) \frac{\sqrt{x_{2}} \beta}{2 \pi}\right] \operatorname{cosec} \frac{\sqrt{x_{2}} \beta}{2}\right. \\
& \left.\left.+\frac{2 \pi^{2}}{x_{2} \beta^{2}}\right\}\right]+\frac{F \beta^{2}}{4 \pi^{2}}\left[-\frac{4 \pi^{2}}{x_{3} \beta^{2}}+2\left\{-\frac{\pi^{2}}{\sqrt{x_{3} \beta}}\right.\right. \\
& \left.\left.\times \cos \left[\left(\pi-\frac{4 \pi \tau_{0}}{\beta}\right) \frac{\sqrt{x_{3}} \beta}{2 \pi}\right] \operatorname{cosec} \frac{\sqrt{x_{3}} \beta}{2}+\frac{2 \pi^{2}}{x_{3} \beta^{2}}\right\}\right] .
\end{aligned}
$$

В результате аналитически найдено выражение для вероятности одномерного туннельного переноса:

$$
\Gamma=B \exp (-S) .
$$

\section{Благодарности}

Авторы благодарят проф. Э.Дж. Леггета за полезные обсуждения, а также ИПЛИТ РАН, ЦКП МГУ им. М.В. Ломоносова и НОЦ „Физика твердотельных наноструктур“ ННГУ им. Н.И. Лобачевского за помощь при выполнении экспериментальной части работы.

\section{Финансирование работы}

Работа выполнена при поддержке гранта Министерства науки и высшего образования РФ 0748-2020-0012 и гранта РФФИ 18-42-130007 p_a. The International Center for Interfacing Magnetism and Superconductivity with Topological Matter MagTop is supported by the Foundation for Polish Science through the IRA Programme co-financed by EU within SG OP (Grant No. MAB/2017/1).

\section{Конфликт интересов}

Авторы заявляют, что у них нет конфликта интересов.

\section{Список литературы}

[1] A.O. Caldeira, A.J. Leggett. Ann. Phys., 149 (2), 374 (1983).

[2] A.I. Larkin, Yu.N. Ovchinnikov. Phys. Rev. B, 28 (11), 6281 (1983).

[3] B.I. Ivlev, Yu.N. Ovchinnikov. J. Exp. Theor. Phys., 66 (2), 378 (1987). [Zhurnal Éksperimental'noǐ i Teoreticheskoř Fiziki, 93, 668 (1987).]

[4] A.K. Aringazin, Yu.I. Dahnovsky, V.D. Krevchik, M.B. Semenov, A.A. Ovchinnikov, K. Yamamo. Phys. Rev. B, 68, 155426 (2003).

[5] Yu.N. Ovchinnikov. J. Exp. Theor. Phys., 104 (2), 254 (2007). https://doi.org/10.1134/S1063776107020100
[6] V.A. Benderskii, E.V. Vetoshkin, H.P. Trommsdorff, E.I. Kats. Phys. Rev. E, 67, 026102 (2003).

[7] Transfer Processes in Low-Dimensional Systems (in memory of A.A. Ovchinnikov, A.I. Larkin), ed. by A.J. Leggett, Yu. Dahnovsky, V.D. Krevchik, V.Ya. Krivnov, M.B. Semenov, K. Yamamoto (UT Research Institute Press, Tokyo, Japan, 2005)

[8] V.Ch. Zhukovsky, Yu.I. Dahnovsky, O.N. Gorshkov, V.D. Krevchik, M.B. Semenov, Yu.G. Smirnov, E.V. Chuprunov, V.A. Rudin, N.Yu. Skibitskaya, P.V. Krevchik, D.O. Filatov, D.A. Antonov, M.A. Lapshina, M.E. Shenina, K. Yamamoto. Moscow Univ. Phys. Bull., 64 (5), 475 (2009). https://doi.org/10.3103/S0027134909050014

[9] V.Ch. Zhukovskii, O.N. Gorshkov, V.D. Krevchik, M.B. Semenov, E.V. Groznaya, D.O. Filatov, D.A. Antonov, Moscow Univ. Phys. Bull., 64 (1), 27 (2009). https://doi.org/10.3103/S0027134909010068

[10] D. Filatov, D. Guseinov, I. Antonov, A. Kasatkin, O. Gorshkov. RSC Adv., 4, 57337 (2014).

[11] Управляемое диссипативное туннелирование. Туннельный транспорт в низкоразмерных системах, под ред. Э.Дж. Леггета, А.К. Арынгазина, В.А. Бендерского, Ю.И. Дахновского, Х. Деккер, В.Ч. Жуковского, В.Д. Кревчика, Ю.Н. Овчинникова, М.Б. Семенова, А.И. Тернова, К. Ямамото (Физматлит, М., 2011-2012)

[12] F.V. Kusmartsev, V.D. Krevchik, M.B. Semenov, D.O. Filatov, A.V. Shorokhov, A.A. Bukharaev, Y. Dakhnovsky, A.V. Nikolaev, N.A. Pyataev, R.V. Zaytsev, P.V. Krevchik, I.A. Egorov, K. Yamamoto, A.K. Aringazin. J. Exp. Theor. Phys. Lett., 104, 392 (2016). https://doi.org/10.1134/S0021364016180016

[13] Н.Н. Леденцов, В.М. Устинов, В.А. Щукин, П.С. Копьев, Ж.И. Алферов, Д. Бимберг. ФТП, 32 (4), 385 (1998).

[14] O. Stier, M. Grundmann, D. Bimberg. Phys. Rev. B, 59 (8), 5688 (1999).

[15] I.A. Karpovich, B.N. Zvonkov, N.V. Baidus' et al. Trends in Nanotechnology Research (Nova Science Publishers, NY., 2004) 